EPJ Web of Conferences 81, 05024 (2014)

DOI: $10.1051 /$ epjconf/20148105024

(C) Owned by the authors, published by EDP Sciences, 2014

\title{
New results on Coulomb effects in meson production in relativis- tic heavy ion collisions
}

\author{
Andrzej Rybicki ${ }^{1, a}$, Antoni Szczurek ${ }^{1,2}$, and Mariola Kłusek-Gawenda ${ }^{1}$ \\ ${ }^{1}$ H.Niewodniczański Institute of Nuclear Physics, Polish Academy of Sciences, Radzikowskiego 152, 31-342 \\ Kraków, Poland \\ ${ }^{2}$ University of Rzeszów, Rejtana 16, 35-959 Rzeszów, Poland
}

\begin{abstract}
We propose a new method of investigating the space-time evolution of meson production in heavy ion collisions, by making use of spectator-induced electromagnetic ("Coulomb") effects. The presence of two nuclear remnants ("spectator systems") in the non-central collision generates a strong Coulomb field, which modifies the trajectories of charged final state hadrons. This results in charge-dependent azimuthal anisotropies in final state meson emission. In our approach, this effect can be computed numerically by means of a high-statistics Monte Carlo simulation, using the distance between the meson formation zone and the spectator system as free parameter. Our simulation correctly describes the electromagnetic effect on azimuthal anisotropies observed for $\pi^{+}$and $\pi^{-}$ mesons in Au+Au collisions at lower RHIC energy, known from data recently reported by the STAR Collaboration. Similarly to our earlier studies of spectator-induced electromagnetic effects, also in the present study we find that these effects offer sensitivity to the position of the meson formation zone with respect to the spectator system. Therefore, we conclude that they can serve as a new tool to investigate the space-time evolution of meson production, and the dynamics of the heavy ion collision.
\end{abstract}

\section{Introduction}

In relativistic heavy ion collisions in the energy regime of at least a few $\mathrm{GeV}$ per participating nucleon pair, two main elements of the heavy ion reaction are differentiated. These are the system created by the nucleons participating directly to the collision (the "participant zone") and the nuclear remnants that do not take direct part in the reaction (the two "spectator systems"). The net positive charge of the spectator systems induces a sizeable Coulomb field in their respective rest frames. In the collision center-of-mass system, this Coulomb field $\left(\vec{E}^{\prime}\right)$ transforms into a full electromagnetic field consisting of both electric and magnetic components $(\vec{E}, \vec{B})$. Classically we obtain [1]:

$$
\begin{aligned}
\vec{E} & =\gamma \vec{E}^{\prime}-\frac{\gamma^{2}}{\gamma+1} \vec{\beta}\left(\vec{\beta} \cdot \vec{E}^{\prime}\right), \\
\vec{B} & =\gamma\left(\vec{\beta} \times \vec{E}^{\prime}\right),
\end{aligned}
$$

\footnotetext{
ae-mail: andrzej.rybicki@ifj.edu.pl
} 
where $\vec{\beta}$ is the velocity vector of a given spectator system and $\gamma=\left(1-\beta^{2}\right)^{-\frac{1}{2}}$. As a result, each charged particle created in the collision is subjected to a summed electromagnetic field which can modify its trajectory. The magnitude of this field depends on time as well as on the position of the particle with respect to the two spectator systems.

In our earlier works [1,2], we studied the effect of this electromagnetic field on the final state spectra of charged mesons, and in particular $\pi^{+} / \pi^{-}$and $K^{+} / K^{-}$ratios. We found that this effect is sensitive to the initial conditions imposed on pion and kaon emission, and therefore brings new information on the space-time evolution of charged meson production. More recently, we studied the influence of the above electromagnetic field on the "directed flow" of charged pions. Directed flow in heavy ion collisions is subject of intensive studies as it is supposed to reflect collective phenomena occurring in the collision; specifically, it reflects the collective sidewards motion of the emitted particles. It is defined as the first Fourier coefficient of the azimuthal distribution of the particles with respect to the reaction plane [3]:

$$
v_{1} \equiv\left\langle\cos \left(\phi-\Psi_{\mathrm{RP}}\right)\right\rangle,
$$

where $\phi$ and $\Psi_{\mathrm{RP}}$ denote the azimuthal angle of the emitted particle and of the orientation of the reaction plane, respectively. Our analysis of charged pion directed flow in $\mathrm{Pb}+\mathrm{Pb}$ collisions [4] demonstrated that the spectator-induced electromagnetic effect results in very large absolute values of $v_{1}$ for positive pions at target and beam rapidities, in agreement with data from the WA98 experiment [5]. This suggests the emission site of these pions to be placed relatively close to the corresponding spectator system, at a longitudinal distance $d_{E}$ below $1 \mathrm{fm}$.

In the present paper we focus on the electromagnetic effect which the spectator systems induce on positive and negative pions in the region of central rapidities, in the context of the new experimental data provided by the STAR Collaboration [6].

\section{Charge-dependent azimuthal anisotropy in pion emission}

The azimuthal anisotropy in the emission of positive and negative pions in the final state of the $\mathrm{Au}+\mathrm{Au}$ collision at the energy $\sqrt{s_{N N}}=7.7 \mathrm{GeV}$ is presented in figure 1 . This anisotropy is quantified by the directed flow $v_{1}$ (equation 2), and shown as a function of the reduced rapidity of the pion. For both pion charges, the rapidity dependence of $v_{1}$ exhibits a common characteristic behaviour of a smooth decrease with a passage through zero at central rapidity. This behaviour is well known from measurements of summed charged pions in a broad range of collision energies up to the LHC regime

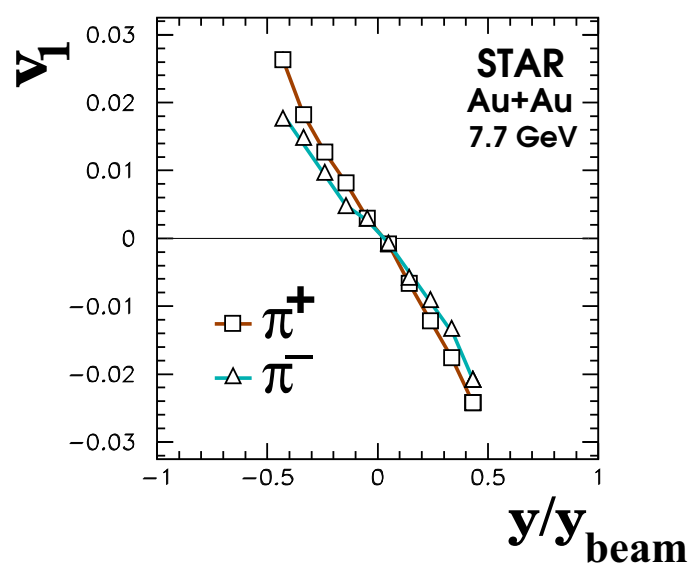

Figure 1. Directed flow of positive and negative pions in intermediate centrality $(10-40 \%) \mathrm{Au}+\mathrm{Au}$ collisions at $\sqrt{s_{N N}}=7.7 \mathrm{GeV}$, drawn as a function of the pion reduced rapidity $y / y_{\text {beam }}$ (in the collision c.m.s.). This result was obtained by the STAR experiment at RHIC; the data points are redrawn from [6]. Lines serve to guide the eye. 
(see, e.g., [7, 8]). However, at the same time, a charge splitting between positive and negative pions is evident. This indicates that an additional effect of repulsion of positive and attraction of negative pions takes place on top of their well-known common behaviour.

As such we assume that the total value of the pion directed flow can be approximated by the sum of two components, one $\left(v_{1}^{\text {flow }}\right)$ coming from the strong and the other $\left(v_{1}^{\pi^{ \pm}, E M}\right)$ coming from the electromagnetic interaction:

$$
\begin{aligned}
& v_{1}^{\pi^{+}} \approx v_{1}^{\text {flow }}+v_{1}^{\pi^{+}, E M}, \\
& v_{1}^{\pi^{-}} \approx v_{1}^{\text {flow }}+v_{1}^{\pi^{-}, E M} .
\end{aligned}
$$

From our earlier studies [4] we know that $v_{1}^{\pi^{+}, E M} \approx-v_{1}^{\pi^{-}, E M}$ in the considered range of rapidities. On that basis we can directly extract the values of $v_{1}^{\pi^{+}}, E M$ and $v_{1}^{\pi^{-}, E M}$ from equation 3 . This electromagnetic component of pion directed flow is shown as data points in figure 2.

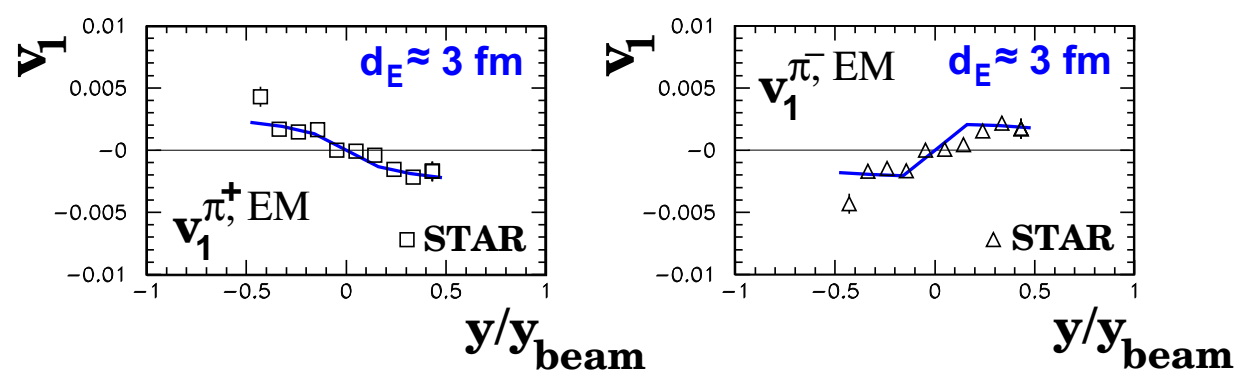

Figure 2. Electromagnetic component of directed flow of positive (left) and negative (right) pions. The values extracted from experimental measurements (equation 3) are shown as data points; these are compared to our model simulation described in the text and shown as the solid curve.

We now turn towards the numerical simulation of the spectator-induced electromagnetic interaction. For this purpose we use a very simple model, succesfully applied before for the description of spectator-induced electromagnetic distortions of $\pi^{+} / \pi^{-}$ratios in $\mathrm{Pb}+\mathrm{Pb}$ collisions [2]. An in-depth description of this model can be found in $[1,4]$. We approximate the two spectator systems as homogenously charged spheres moving at the proper relativistic velocity. The Coulomb field of each of the two spheres transforms into the full electromagnetic field as given by equation 1 . We assume a point-like source and a single time for the emission of the sample charged pions which are then numerically propagated through the electromagnetic field. The point-like source is placed at the original interaction point. The initial conditions imposed on this simplified model (collision geometry, spectator charge) are carefully adjusted to fit the characteristics of the 10-40\% centrality data sample presented in figure 1. A scaled two-dimensional parametrization of $\left(x_{F}, p_{T}\right)$ spectra of pions measured in nucleon-nucleon collisions [9] is used to define the initial $\pi^{+}$and $\pi^{-}$kinematic distributions before the action of the electromagnetic field. Full azimuthal symmetry is assumed for initial pion emission. Relativistic effects on the EM field (Lorentz contraction, retardation) are taken into account.

A unique free parameter exists in the model, which is the distance $d_{E}$ between the pion emission site and the longitudinal $(z)$ position of each of the two spectator systems. Our model provides a very satisfactory description of the data points in figure 2, provided that a distance of $d_{E} \approx 3 \mathrm{fm}$ is assumed. We note that the result of our electromagnetic simulation is strongly sensitive to $d_{E}$ which appears, as a consequence, to be constrained by the data. Thus we conclude that the measurement 
of charge splitting of directed flow presented in figures 1,2 constitutes a new source of information on the evolution of pion emission in position space as well as in time in the $\mathrm{Au}+\mathrm{Au}$ reaction, once electromagnetic effects are properly taken into account.

\section{Conclusions}

The study presented in the previous Section demontrates that the electromagnetic effects on directed flow of charged pions can be used to obtain information on the distance $d_{E}$ between the pion emission site and the spectator system. Putting the result of this simplified study $\left(d_{E} \approx 3 \mathrm{fm}\right)$ in the context of our earlier analysis of pion directed flow at target and beam rapidities (where a value of $d_{E}$ below $1 \mathrm{fm}$ could be deduced from the data [4]), we note that the obtained effective value of $d_{E}$ decreases with increasing c.m.s. rapidity of the pion. This, as schematically illustrated in figure 3 , reflects the longitudinal evolution of the system created in the course of the heavy ion collision.

We therefore conclude that the elaboration of a new method of investigating the space-time properties of this non-perturbative system is possible, by making use of spectator-induced electromagnetic ("Coulomb") effects. It can be expected that this new method will provide new information on the space-time evolution of pion production, completely independent from other sources such as pion interferometry.

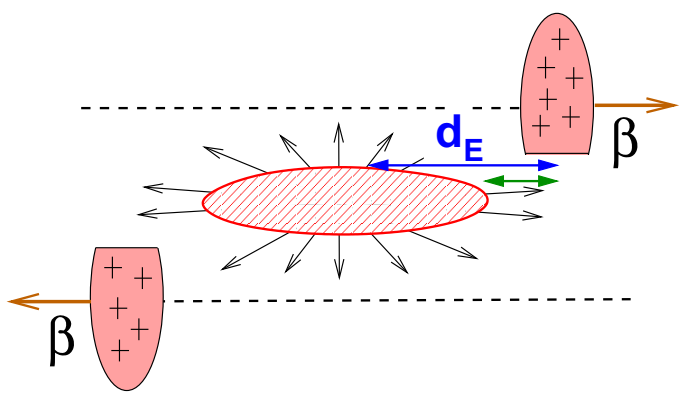

Figure 3. Schematic illustration of the expanding system created in the heavy ion collision. The distance $d_{E}$ between the pion emission site and the spectator decreases with increasing rapidity of the emitted pion, which reflects the longitudinal evolution of the system. Thus, by studying $d_{E}$ as a function of pion rapidity, new information on the spatial extent and on the longitudinal evolution of the system can be obtained.

\section{Acknowledgements}

The authors warmly thank Yadav Pandit and the STAR Collaboration for providing the numerical values for the published STAR data. This work was supported by the Polish National Science Centre (on the basis of decision no. DEC-2011/03/B/ST2/02634).

\section{References}

[1] A. Rybicki, A. Szczurek, Phys. Rev. C 75, 054903 (2007), and references therein.

[2] A. Rybicki, Acta Phys. Polon. B 42, 867 (2011).

[3] A. M. Poskanzer and S. A. Voloshin, Phys. Rev. C 58, 1671 (1998).

[4] A. Rybicki and A. Szczurek, Phys. Rev. C 87, 054909 (2013), and references therein.

[5] H. Schlagheck (WA98 Collaboration), Nucl. Phys. A 663, 725 (2000).

[6] L. Adamczyk et al. (STAR Collaboration), Phys. Rev. Lett. 112, 162301 (2014).

[7] C. Alt et al. (NA49 Collaboration), Phys. Rev. C 68, 034903 (2003).

[8] B. Abelev et al. (ALICE Collaboration), Phys. Rev. Lett. 111, no. 23, 232302 (2013).

[9] C. Alt et al. (NA49 Collaboration), Eur. Phys. J. C 45, 343 (2006). 